\title{
Kerion celsi caused by microsporum gypseum: report of two cases and review
}

\begin{abstract}
Tinea capitis is the scalp infection caused by dermatophyte-mainly by Trichophyton spp and Microsporum spp. The causal agent varies according to the geographic region, the most frequent in the world is M. canis and in the United States of America T. tonsurans. The inflammatory clinical manifestatitions called Kerion Celsi. It is caused mainly by zoophilic fungi as Microsporum canis and Trichophyton mentagrophytes, and rarely by geophilic fungi M. gypseum. We report the case of a 3year-old female patient with an inflammatory tinea capitis with the presence of crusts, zig-zag and comma hairs under trichoscopic examination; a parasited hair was observed in the histopathological study, and Microsporum gypseum was isolated on Sabouraud dextrose agar. In the second case, a 13year-old male patient, the trichoscopy showed "Morse code" hairs and hyaline hyphae and spores were observed in the direct mycological examination. Culture on Sabouraud agar confirmed the presence of Microsporum gypseum. He was treated with Itraconazole for 6 weeks with a complete clinical and mycological resolution.
\end{abstract}

Keywords: inflammatory tinea, kerion celsi, microsporum gypseum, pseudo alopecia, gomori grocott, microconidia
Volume 2 Issue 3 - 2018

Itzel A Flores Reyes,' Diana CVega, ${ }^{2}$

León Felipe Ruiz Arriaga, ${ }^{2}$ Brianda I Uribe

Camacho, ${ }^{2}$ Ana C Franco Marín, ${ }^{3}$ Roberto

Arenas Guzmán ${ }^{4}$

IInternal Medicine Resident, Hospital General Dr. Manuel Gea González, México

2Mycologist, Sección de Micología, Hospital General Dr. Manuel Gea González, México

${ }^{3}$ Resident of Dermatology, Hospital General Dr. Manuel Gea González, México

${ }^{4}$ Dermatologist and Chief of the Mycology Section, Hospital General Dr. Manuel Gea González, México

Correspondence: Roberto Arenas, Dermatologist and Chief of the Mycology Section, Hospital General "Dr. Manuel Gea González". Calzada de Tlalpan 4800, Col. Sección XVI, Del. Tlalpan, Ciudad de México, CP I4080, México, Email rarenas98@hotmail.com

Received: April 09, 2018 | Published: June 14, 2018
Abbrevations: DMSO, dimethylsulfoxide; PCR, polymerase chain reaction; PAS, periodic acid schiff

\section{Introduction}

Dermatophytosis (ring worm) is the most common fungal infection worldwide, affecting $20-25 \%$ of the population, with the highest incidence in the prepubertal age. ${ }^{1,2}$ Tinea capitis is a condition that predominates in rural or suburban areas, representing $4-10 \%$ of all dermatophytosis; affects both sex, with a predominance in females. It is associated with a low socioeconomic status. ${ }^{1}$ It is an almost exclusive infection of preschool and school age children (98\%), due to changes in sebaceous secretion and skin $\mathrm{pH}$ during puberty. ${ }^{1,3}$ Dermatophytes are a group of filamentous fungi that infect keratin-rich tissue. ${ }^{2}$ They are classified into anthropophilic, zoophilic and geophilic species. The most frequent isolated dermatophytes are: Microsporum canis $(80 \%)$, Trichophyton tonsurans $(15 \%)$, other species $(5 \%)$-Trichophyton violaceum, Trichophyton rubrum, $T$. mentagrophytes and Microsporum gypseum. ${ }^{2}$ Currently, $T$. rubrum is the main pathogen in skin and nail infections; while M. canis, $T$. tonsurans and $T$. violaceum predominate on the scalp. ${ }^{2}$

Tinea capitis can be classified clinically as dry (90\%) and inflammatory or Kerion (10\%). ${ }^{1}$ Kerion Celsi is a dermatophytosis caused by zoophilic species such as M. canis, T. mentagrophytes, and, less frequently, M. gypseum -a geophilic dermatophyte. ${ }^{4,5}$ The term Kerion was implemented around $30 \mathrm{a}$. C in Rome by Cornelius Celsi, meaning 'honeycomb' in Greek. Trichophyton tonsurans and Microsporum canis were described as causal agents in 1845 by Hendrick Malsten, and in 1908 by Whitfield, respectively. ${ }^{6}$

\section{Clinical case I}

A 3year-old female from San Miguel de Allende, Guanajuato, Mexico presented with a localized, unilateral, asymmetric dermatosis that affects the scalp in the vertex region, consisting of a pseudoalopecia plaque of approximately $8 \mathrm{~cm} \times 4 \mathrm{~cm}$, with irregular edges, well defined, erythematous base, and meliceric pustules and crusts on the surface, giving the appearance of a honeycomb. Long term progression and barely painful. Without retroauricular adenopathies (Figure 1).

She was previously treated with fluconazole, ketoconazole, topical antiseptics, terbinafine, and cefixime. The diagnosis of microscopic tinea was integrated, so Wood lamp skin examination was performed, showing yellow-green fluorescence. Trichoscopy showed black dots and short hairs, erythema, meliceric crust and zigzag hairs (Figure 2). In a direct examination stained with clorazol black $\mathrm{E}$ was observed an ecto-endothrix parasitism, as well as in the biopsy stained with PAS and Gomori Grocott (Figures 3). The culture in Sabouraud agar showed some powdery white and brown colonies, flat, on the reverse of the culture without pigment. In the microscopical examination of the culture we observed thin-walled, rounded ends with less than six septum macroconidia, and few microconidia (Figure 4).

\section{Clinical case 2}

A 13year-old boy presented with a 15day history of an indurated, erythematous scaling plaque with pustules, golden-yellow crusts and an alopecia patch with well-defined edges on the temporal region of the scalp (Figure 5). Asymptomatic and not previously treated. The scalp dermoscopy showed multiple white bands across the 
hair shaft, called 'Morse code-like' hairs (Figure 6). A superficial scraping of the lesion was performed for direct examination with a potassium hydroxide solution, where hyaline hyphae and spores were visualized (Figure 7). Sabouraud culture showed a powdery beige colony, suggestive of Microsporum gypseum that was confirmed by microscopic examination (Figure 8). Microscopy showed fusiform and thin-walled macroconidia with less than 6septum, arranged in groups and some microconidia (Figure 9). The patient was treated with Itraconazole for 6 weeks, with complete clinical and mycological resolution.

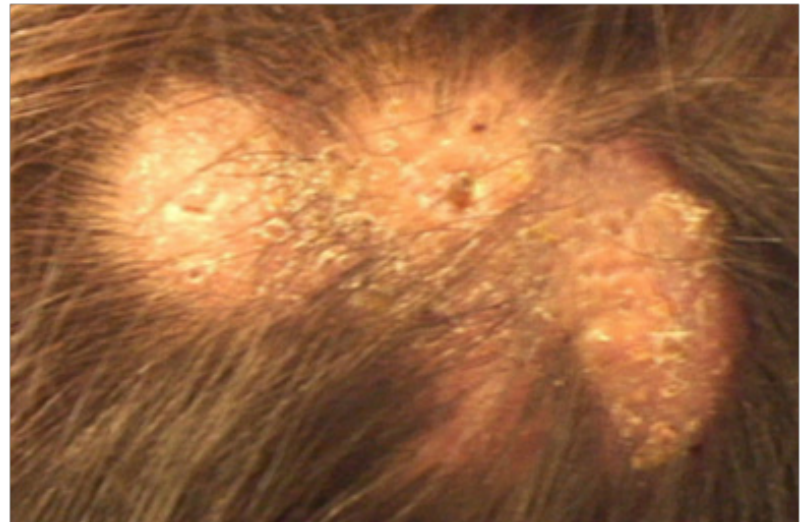

Figure I Kerion Celsi.

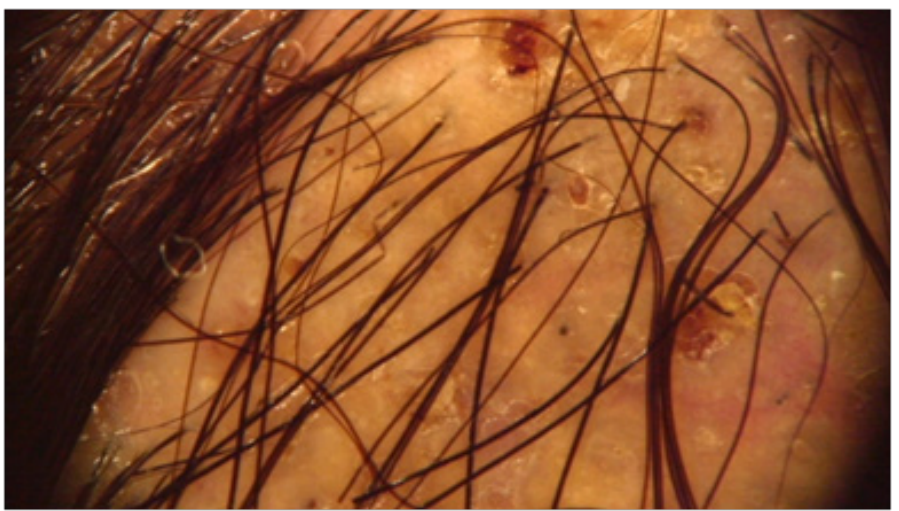

Figure 2 Trichoscopy, zigzag hairs and crusts.

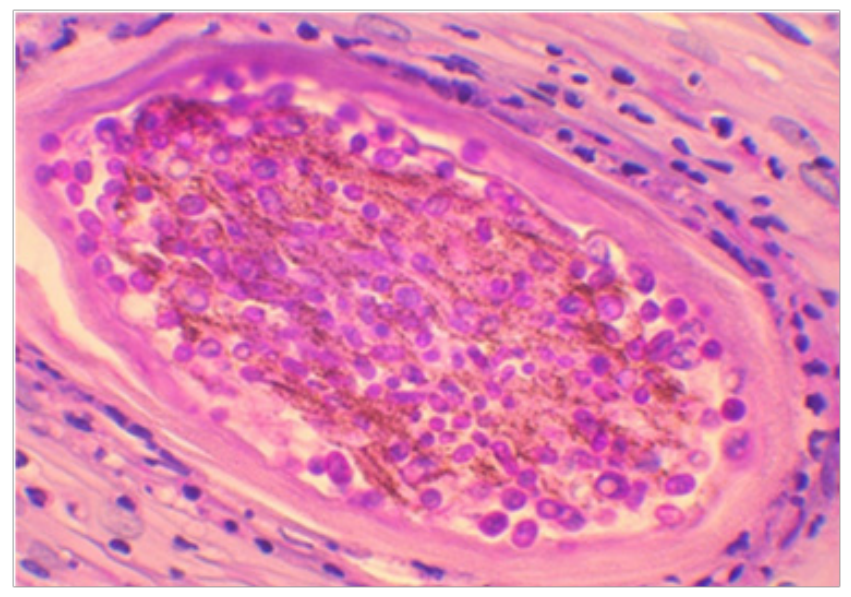

Figure 3 Histopathology, hair with spores (PAS40x).

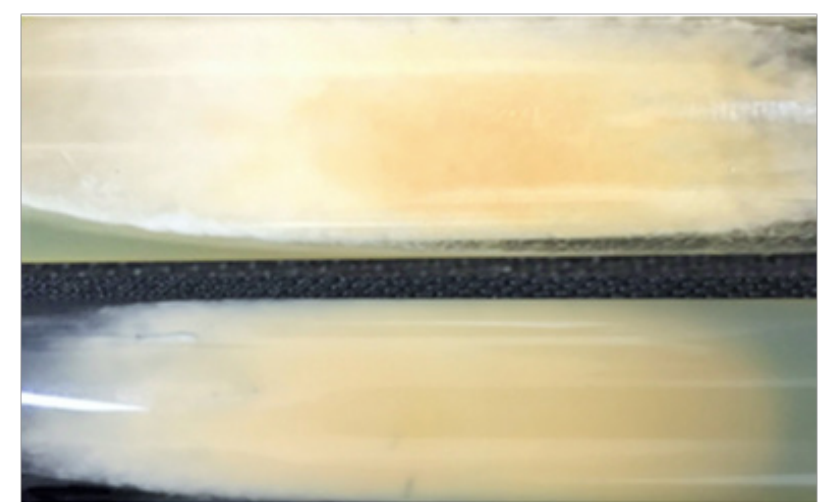

Figure 4 Sabouraud dextroseagar culture, Microsporumgypseum.

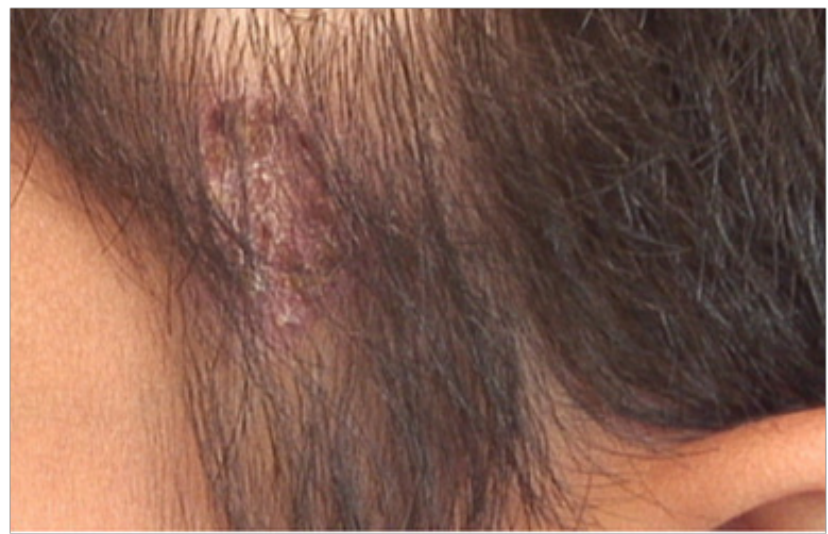

Figure 5 Kerion Celsi.

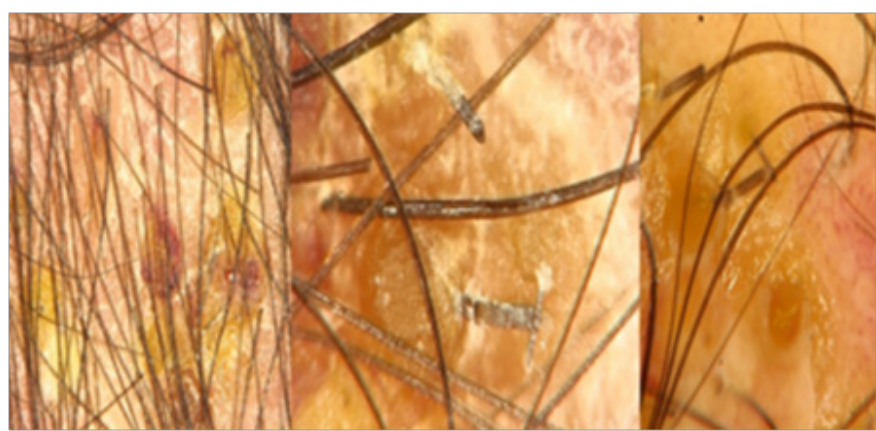

Figure 6 Trichoscopy multiple white bands across the hairs haft called 'Morsecode' hairs.

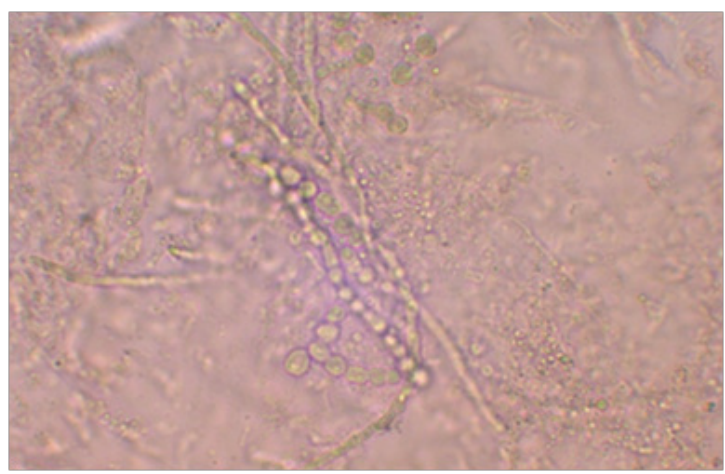

Figure 7 Direct exam hyalinehyphaeandspores. 


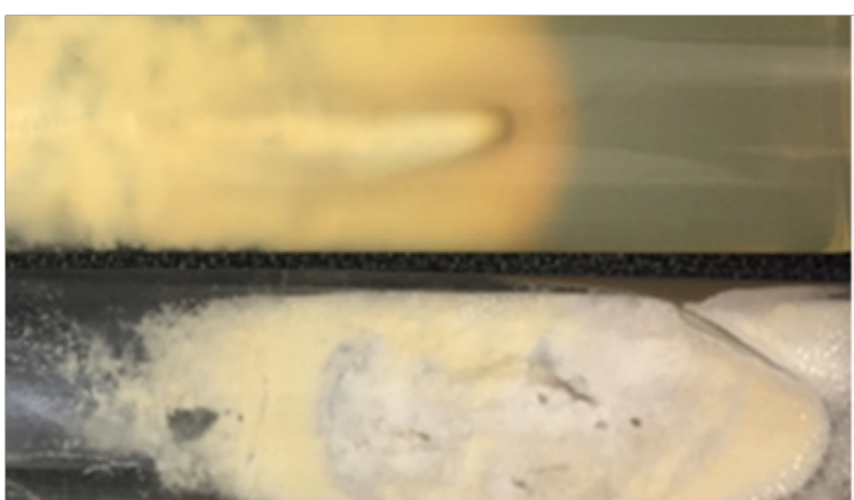

Figure 8 Sabouraud dextroseagar culture, Microsporumgypseum.

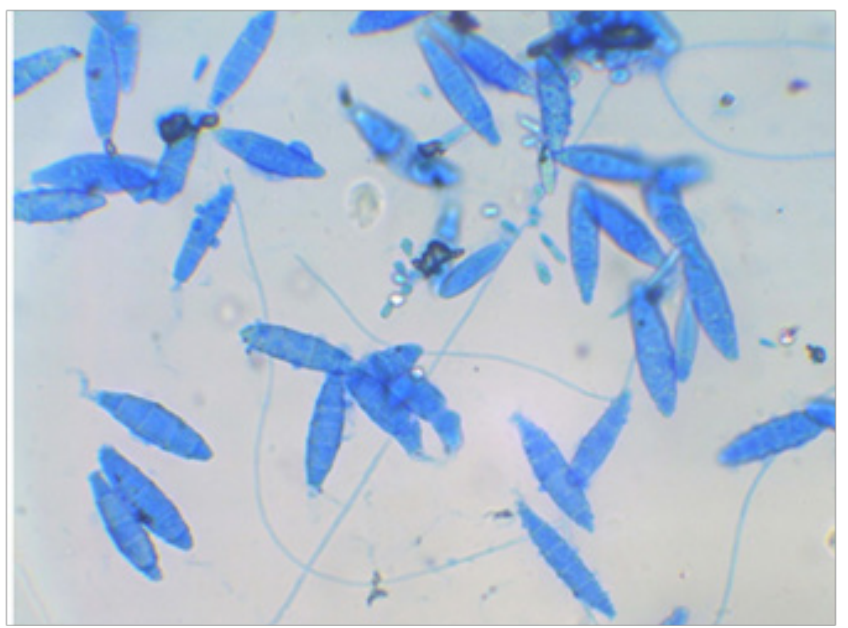

Figure 9 Microscopy image Fusiform and thin walled macro conidia with less than 6septum, arranged in groups, and some micro conidia.

\section{Review}

Epidemiology varies according to the region, changes in lifestyle, nutritional status, low socioeconomic status, poor hygiene, population mobility (migration or immigration), and in recent years, in women has been associated with the postmenopausal age and pregnancy. ${ }^{6}$ It is important to mention that the etiological pattern may change over time, as it has done in the last 30years. ${ }^{7}$ The most affected ages are between 3-7years. ${ }^{5}$ Worldwide, the most frequent agents are $M$. canis and T. tonsurans, the latter with greater spread. ${ }^{8}$ Microsporum gypseum stands out among the infrequent agents worlwide. ${ }^{9}$

In Mexico, dry variety of tinea capitis is present in $90 \%$ of the cases and the inflammatory one in $10 \%$; in $80 \%$ of the cases $M$. canis is isolated, and in less than $1 \%$ M. gypseum..${ }^{10}$ Cases in adults are rare, explained by the antifungal properties of the sebum of the scalp. ${ }^{11}$ Children and prepuberes are predisposed because the lack of sebum, rich in fatty acids and an acid $\mathrm{pH}$, that facilitates colonization and infection by dermatophytes. Short hair has also been associated with higher incidence since spores have an easier access to the scalp. ${ }^{5}$ Immunosuppression is a predisposing factor caused by oncological (leukemia), chronic-degenerative processes (Diabetes mellitus, anemia, etc.), immunosenesence due to elderly, organ transplantation, and use of immunosuppressive drugs. ${ }^{3}$ Immunosuppressants can interfere with hair production and shaft strength, allowing colonization5. Glucocorticoids alter the delayed-type hypersensitivity and generate a The response that increases the chronicity of the Disease. ${ }^{11}$ Tinea capitis is uncommon in patients with HIV, due to increased colonization by Malassezia spp that competitively inhibits colonization by dermatophytes. ${ }^{5}$

In Mexico M. canis and T. tonsurans are the most prevalent etiological agents, compared to the United States, where $T$. tonsurans prevails. ${ }^{5}$ M. gypseum is the most common geophilic dermatophyte, it is very frequent in hands and infrequent in the head; it is acquired by exposure and contact with skin areas exposed to the open air or ground. ${ }^{12}$ Their identification is faster and more accurate through the use of PCR, but the mycological analysis is also important. ${ }^{13}$ The skin has innate defense mechanisms that prevent the growth of dermatophytes (local destruction by neutrophils and macrophages in $60 \%$ and $20 \%$ respectively, presence of b-defensins, cathelicidin LL$37 \mathrm{~m}$, and dermicidin. On the scalp polyunsaturated medium-chain fatty acids also play a protective role.

If any of these protective factors is altered, the skin is prone to infection. First there is a fungal transfer from the environment, then there is an invasion of the skin or hair follicles of arthrospores that adhere to the keratinocytes in an average time of 2-3hours, and at the same time proteolytic enzymes production. Resulting in a noninflammatory or severe inflammatory process. ${ }^{8}$ When a conidium reaches the scalp, it develops a mycelium that grows, reaches the hair infundibulum, descends in its corneal lining and penetrates the hair; It descends through the hair bulb to the keratinized area known as the Adams line. The non-affected pillar papilla continues to elaborate the hair that grows normally but emerges parasitized, and therefore loses its normal structure and breaks a few millimeters from the cutaneous surface. ${ }^{1}$

According to the pattern of fungal invasion, it can be classified as an endothrix, with 2 varieties (trichophytic and favic), and the ecto-endothrix type with 3varieties-microsporic, microide, and megasporada. This depends on the clinical manifestations as well as the amount of inoculum and the immunological status of the patient. The following table shows the type of invasion, its description, the most frequent associated agent, the clinical manifestations, and the Wood lamp skin examination ${ }^{8}$ (Table 1).

Clinical clasification depends on the etiological agent, the immune status of the host, the type of invasion, and the degree of inflammation (inflammatory and non-inflammatory). In the inflammatory presentation there are soft plaques covered with broken hairs and pustules, which is classified into 6subtypes ${ }^{6}$ (Table 2). In the noninflammatory presentation there is flaking, hair loss, and seborrhea; ectothix is presented with circumscribed alopecia patches. ${ }^{8}$ The common characteristic among the different etiologies is the variable loss of hair with some degree of inflammation. Pruritus may be present; hair loss (with or without fracture) and scale (focal or diffuse) are the most representative signs. ${ }^{7}$ The presence of cervical and suboccipital lymphadenopathies can guide the diagnosis. ${ }^{5}$

The spectrum of the disease varies from some broken hairs with small scales, to a very painful inflammatory plaque with purulent discharge and the presence of lymphadenopathies (Kerion). ${ }^{8}$ There may be extensive and uneven hair loss, with scars and atrophy. Secondary bacterial infection is frequent. Some characteristics have been found depending on the etiology but are not considered specific. 
In the infection by $M$. canis there is great inflammation and pruritus; in $T$. tonsurans and $T$. violaceum there is minimal inflammation with hair loss in multiple patches with few scales and presence of blackheads; in T. verrucosum or T. mentagrophytes a painful tumor with inflammation and lymphadenopathy may be present. ${ }^{8}$ The diagnosis is based on clinical suspicion mainly, but there are several diagnostic aids such as dermoscopy, direct microscopy, culture, and Wood lamp skin examination John et al. Propose the diagnostic criteria that are presented in the following (Tables 3-5).

Table I Tinea capitis: types of invasion

\begin{tabular}{|c|c|c|c|c|}
\hline $\begin{array}{l}\text { Type of } \\
\text { invasion }\end{array}$ & Description & Frequent agent & Clinic & Wood's light \\
\hline \multirow[t]{2}{*}{ Ectothrix } & $\begin{array}{l}\text { The hair shaft is invaded at the } \\
\text { level of mid-follicle. The }\end{array}$ & Microsporum & $\begin{array}{l}\text { Gray patch with circular alopecia and scale. } \\
\text { Inflammation and rupture of the follicles } \\
2-3 \mathrm{~mm} \text { above the skin. }\end{array}$ & Fluorescence. \\
\hline & $\begin{array}{l}\text { intrapilary hyphae grow down } \\
\text { towards the bulb of } \\
\text { the hair. }\end{array}$ & & & \\
\hline Endothrix & Spores inside the hair shaft. & $\begin{array}{l}\text { T. tonsurans, T. soudanese } \\
\text { and members of T. rubrum } \\
\text { and T. violaceum. }\end{array}$ & $\begin{array}{l}\text { Hairs broken at the level of the skin (black } \\
\text { points). }\end{array}$ & No fluoresce. \\
\hline \multirow[t]{2}{*}{ Favus } & $\begin{array}{l}\text { Yellowish traces in the hair cortex } \\
\text { that contain hyphae forming a } \\
\text { crusting with white scales. }\end{array}$ & T.schoenleinii. & $\begin{array}{l}\text { Air spaces in the hair follicles (the presence } \\
\text { of hyphae that form groups in their entrance } \\
\text { to the epidermis). Hairs with less damage } \\
\text { than the former two types, and can continue } \\
\text { to grow in length. }\end{array}$ & Variable. \\
\hline & $\begin{array}{l}\text { Hyphae and air bubbles inside the } \\
\text { hair shaft. }\end{array}$ & & & \\
\hline
\end{tabular}

Table 2 Types of Inflammatory dermatophytic infections

\begin{tabular}{|c|c|}
\hline Type of inflammatory tinea & Characteristics \\
\hline Pustular & Irregular alopecia and scattered pustules or low-grade folliculitis \\
\hline Favus & Erythema around the hair follicles and scarring alopecia \\
\hline Majocchi granuloma & Papular or nodular lesions on the face or extremities \\
\hline Mycetoma & $\begin{array}{l}\text { Nodular lesions that overlap with erythematous and scaly plaques, sinusoid } \\
\text { tracts with purulent drainage and pseudo alopecia }\end{array}$ \\
\hline Scutula & Yellowish crusts with hair loss and scars, may also involve skin and nails \\
\hline kerion & $\begin{array}{l}\text { Usually solitary lesion, frequent in the occipital area of the scalp, although } \\
\text { they can be multiple, painful, with indurated plaques that begin as dry lesions } \\
\text { with scale and short hairs. Depends on the immune status of the host, usually } \\
\text { resolves in } 8 \text { weeks with treatment }\end{array}$ \\
\hline
\end{tabular}

Table 3 Major and minor criteria for diagnosis of Inflammatory tinea capitis (Modified from John A6)

\begin{tabular}{ll}
\hline Major citeria & Minor criteria \\
\hline Tenderness to touch & Dermatophytid reaction \\
Alopecia surrounding the lesion & Regional lymphadenopathy \\
Numerous pustules and purulent drainage & Short hairs on dermoscopy \\
Scaling at the lesion & Boggy plaques \\
& Clear dermarcation of borders \\
& Overlying erythema \\
& Pruritus \\
\hline
\end{tabular}


Table 4 Severity scale, clinic, histology, and description (Modified from John $A^{6}$ )

\begin{tabular}{|c|c|c|c|}
\hline Grade & Clinic & Histology & Description \\
\hline I & $\begin{array}{l}\text { Erythematous plaque covered by } \\
\text { some pustules, most of the hairs are in } \\
\text { catagen phase }\end{array}$ & Suppurative folliculitis & $\begin{array}{l}\text { Inflammatory perifollicular infiltrate with spongiosis and } \\
\text { neutrophilic infiltrate, lymphocytes and with plasma cells, where } \\
\text { most of the follicles are in catagen stage. }\end{array}$ \\
\hline 2 & $\begin{array}{l}\text { Pustules and papukes overlying } \\
\text { erythematous plaque; scaling at the } \\
\text { periphery of plaque, few disrupted hai } \\
\text { follickes }\end{array}$ & $\begin{array}{l}\text { Folliculitis with } \\
\text { suppurative dermatitis }\end{array}$ & $\begin{array}{l}\text { Neutrophilic perivascular and perifolicular inflammatory } \\
\text { infiltrate, with plasma-cell, lymphocytes, and most of the follicles } \\
\text { are in catagen and telogen stage. Only few follicles are affected. }\end{array}$ \\
\hline 3 & $\begin{array}{l}\text { Pustules, papules, scaling and erythema, } \\
\text { with or without vesicles and alopecic } \\
\text { areas }\end{array}$ & $\begin{array}{l}\text { Folliculitis with } \\
\text { granulomatous and } \\
\text { suppurative dermatitis }\end{array}$ & $\begin{array}{l}\text { Suppuration and granulomatous dermatitis, with neutrophilic } \\
\text { infiltrate, lymphocytes, plasma-cells and granulomatous reaction. } \\
\text { There is decreased number of hair follicles. }\end{array}$ \\
\hline 4 & $\begin{array}{l}\text { Pustules, scale, erythema; scarring } \\
\text { alopecia patches }\end{array}$ & $\begin{array}{l}\text { Suppurative and } \\
\text { granulomatous } \\
\text { dermatitis with } \\
\text { fibrosing dermatitis }\end{array}$ & $\begin{array}{l}\text { Inflammatory infiltrate of neutrophils, lymphocytes and plasma } \\
\text { cells with granuloma formation, increase in the number of } \\
\text { collagen fibers, and loss of hair follicles consistent with scarring } \\
\text { alopecia. }\end{array}$ \\
\hline
\end{tabular}

Table 5 Treatment approved oral antifungal agents for treatment of tinea capitis. Dosage recommendations (Modified from Gupta AK ${ }^{8}$ )

\begin{tabular}{|c|c|c|c|}
\hline $\begin{array}{l}\text { Antifungal } \\
\text { agent }\end{array}$ & Children (FDA approved) & Adults (FDA approved) & $\begin{array}{l}\text { Children (British Association } \\
\text { of Dermatologists Guidelines) }\end{array}$ \\
\hline \multirow[t]{3}{*}{ Terbinafine } & $<25 \mathrm{Kg}, 125 \mathrm{mg} /$ day & $250 \mathrm{mg} /$ day, $4-6$ weeks & I50mg/day, 4weeks \\
\hline & $25-35 \mathrm{Kg}, 187.5 \mathrm{mg} /$ day & & \\
\hline & $>35 \mathrm{Kg}, 250 \mathrm{mg} /$ day & & \\
\hline Fluconazole & 3 to $5 \mathrm{mg} / \mathrm{kg} / \mathrm{day}$ & $50 \mathrm{mg} /$ day, $4-7$ weeks or $150 \mathrm{mg} /$ week, 4-8weeks & I50mf/week, 4weeks \\
\hline Itraconazole & $3-5 \mathrm{mg} / \mathrm{kg} /$ day & $100-200 \mathrm{mg} /$ day, 4 weeks & $100 \mathrm{mf} /$ day, 4weeks \\
\hline Griseofulvin & $125-250 \mathrm{mg} / \mathrm{kg} /$ day & & 200-300mg/day, 8weeks \\
\hline ultramicronize & & & \\
\hline
\end{tabular}

The use of dermoscopy in ringworm of the head (Trichoscopy) is becoming more frequent, because is rapid diagnostic method. ${ }^{14,15}$ Coma hairs pattern is classic in endothrix and ectothrix parasitization; there are nonspecific findings such as black dots, broken and zig-zag hairs, as well as morse code hairs. The dermoscopy also supports us in the differential diagnosis between tinea capitis and alopecia areata, the latter is characterized by yellow spots, exclamation hairs and short hairs. ${ }^{8}$ Wood lamp skin examinatiSon is useful as it provides guidance for the identification of the microorganism involved; it will be negative in trichophytic tinea-with the exception of $T$. schoenleinii because the infection is endothrix, it does not produce the characteristic yellowgreenish fluorescence observed with organisms that cause ectoendothrix parasitization like $M$. canis (exhibits green fluorescence), and in tinea capitis favosa (exhibits weak blue fluorescence). ${ }^{5}$

Direct microscopic examination is performed by adding $\mathrm{KOH}+$ dimethylsulfoxide (DMSO) or clorazol black E to the broken hairs. John, Schwartz and Janniger proposed the next major and minor criteria for diagnosis of kerion: Major: tenderness, alopecia, scaling, pustules and purulent drainage; minor criteria: dermatophytid reaction, lymphadenopathy, comma or corkscrew hairs, boggy plaques, clear dermarcation of borders and overlying erythema and pruritus. ${ }^{5}$
The culture of the lesion is performed on Sabouraud agar. It is considered the gold standard to confirm the diagnosis and the ethiological agent, with an average growth time of 2 weeks (with the exception of T. verrucosum, which requires 3 weeks). The use of polymerase chain reaction (PCR) has optimized the waiting time by reporting results in 1day compared to cultures. ${ }^{8}$ It is considered a reliable method and provides better results than the use of cultures because it allows more precise diagnoses when identifying the etiological agent or making differential diagnoses in similar clinical presentations. In addition, it allowed Trichophyton, Epidermophyton, Nannizzia, Microsporum to be classified in the multilocus taxonomic tree. $^{13}$

The histological study is useful in patients with negative cultures, it reveals spores surrounding the hair follicle and hyphae within the follicle. There is an inflammatory infiltrate in the dermis with giant cells and follicles destruction. Periodic acid Schiff (PAS) staining can confirm the diagnosis of Kerion (neutrophilic and granulomatous infiltrate in the early stages and fibrotic scar in the latter); the 4 histological types are presented in the (Table 5) (Table 4), Arenas et al. ${ }^{16}$ conducted a study of histopathological and mycological findings in 19children with inflammatory tinea. In the mycological study 
found that the most frequent etiological agents was M.canis (32\%), T.mentagrophytes $(27 \%)$, T.tonsurans $(21 \%)$, T.rubrum $(10 \%)$, and M.gypseum (5\%); the histopathological findings were: suppurative folliculitis (11\%), suppurative folliculitis with suppurative dermatitis (37\%), suppurative folliculitis with suppurative and granulomatous dermatitis (26\%), and suppurative and granulomatous dermatitis with folliculitis with fibrosing dermatitis $(26 \%)$. In the histopathological study $65 \%$ of the cases presented fungal structures that were stained with PAS and Gromori-Grocott stain. ${ }^{16}$

Differntial diagnosis includes several conditions that are capable of causing alopecia and inflammation of the scalp, the most important are alopecia areata (no epidermal changes, exclamation hais; no crusting, inflammation, or pustules), atopic dermatitis (personal or family history of asthma, fever, sensitive skin; usually no lymphadenopathy; usually no alopecia), bacterial scalp abscess (painful to touch without areas of alopecia), psoriasis (family history of psoriasis; gray or silver scale; other systemic involvement), seborrheic dermatitis (fatty scale with no alopecia or lymphadenopathy), and trichotillomania (no scale and the length of the hairs is variable). ${ }^{5}$ The objective of a successful treatment is based on the relief of symptoms, the evidence of clinical improvement, the eradication of the mycological process and the prevention of transmission. The treatment can be oral and / or topical. ${ }^{7}$ To eradicate tinea capitis, oral antifungal therapy is essential-except for infants, especially in short-course infections which are managed topically-the treatment of choice is Griseofulvin, since it is fungistatic (inhible the synthesis of nucleic acids and interrupts cell division) and anti-inflammatory. Its absorption and biosavailability improve with the intake of fatty foods.

Oral griseofulvin has a higher efficacy rate (88-100\%) compared to terbinafine (34.4-36.5\%), although both drugs depend on the envolved dermatophyte. Terbinafine has a higher cure rate (47.7-56.1\%) for $T$. tonsurans compared to griseofulvin $(23.8 \%-30.6 \%)$. Itraconazole and fluconazole are not approved by the FDA for the treatment of inflammatory ringworm of the head and its use is suggested in patients with evidence of immunosuppression or systemic mycosis. ${ }^{15}$ The time to achieve a complete cure varies. Terbinafine has a lower cure rate compared to griseofulvin ( 4 vs 8 weeks). Short-term treatment is preferred to avoid patient non-compliance. ${ }^{7}$ Chen et al. ${ }^{15}$ carried out a systemic antifungal therapy of tinea capitis in children and concluded that new treatments such as terbinafine, itraconazole and fluconazole have similar effects as griseofulvin in children with trichosporic tinea of the head, while ketoconazole may be less effective than the griseofulvin. ${ }^{15}$ The new evidence suggests that terbinafine is more effective than griseofulvin in children with T. tonsurans; however, in children with Microsporum infections it is suggested that the effect of griseofulvin is better than terbinafine, but also itraconazole is a better option for microsporic infections of the scalp ${ }^{6}$ There is no significant evidence between the use of 4 vs 8 weeks of griseofulvin. Not all treatments for tinea capitis are available in pediatric formulations, but all have a reasonable safety profile ${ }^{15}$ (Table 5 ).

Topical treatment with antifungal shampoo can be used in combination with oral antifungal therapy as a preventive measure to avoid asymptomatic carriers, decrease pruritus and improve appearance. The most used are selenium sulphide 1\% (heavy metal, decreases the number of spores, but with bad smell), Povidone iodine, ciclopirox 1\% (Hydroxypyridone that inhibits the degradation of peroxides in fungi), the last two have not shown differences in the cure rate twice a week. ${ }^{7}$ Using wet compresses can be useful to eliminate exudates. ${ }^{8}$

In severe forms, oral steroids can be used to decrease the inflammatory response. The transmission of tinea capitis is due to direct contact with infected patients, animals or asymptomatic carriers (especially T. tonsurans). Objects such as brushes and hats must be for personal use. Children can freely attend school after 10days of the start of oral treatment to avoid contagion.?

\section{Conclusion}

In Conclusion, Kerion Celsi is an inflammatory state that, paradoxically; and although etiological agents are more frequently of zoophilic origin, this is not related to the aggressive capacity of the dermatophyte. It is essential to establish an early diagnosis and treatment to avoid the consequences of definitive alopecia. In adults, there are no studies on first-line treatment for M. gypseum infections. Almost all dermatophytosis studies are performed in the pediatric population, and not all drugs are approved in all countries.

\section{Acknowledgements}

None.

\section{Conflict of interest}

The author declares no conflict of interest.

\section{References}

1. Rebollo N, López-Barcenas AP, Arenas R. Tiña de la cabeza. Actas Dermsifiologr. 2008;99(2):91-100.

2. Zhan P, Liu W. The Changing Face of Dermatophytic Infections Worldwide. Mycopathologia. 2017;182(1-2):77-86.

3. Arenas R. Dermatofitosis en México. Rev Iberoam Micol. 2002;19:63-67.

4. Baudraz Rosselet F, Bontems O, Ninet B, et al. A Kerion celsi caused by Microsporum gypseum: unusual direct mycological examination. Mycoses. 2006;49(2);145-146.

5. John AM, Schwartz RA, Janniger CK. The kerion: an angry tinea capitis. Int J Dermatol. 2018;57(1):3-9.

6. Arenas R. Micologia Medica Ilustrada. 5th ed. Mexico: McGraw-Hill; 2014: 67-98.

7. Gupta AK, Foley KA, Versteeg SG. New Antifungal Agents and New Formulations Against Dermatophytes. Mycopathologia. 2017;182:127141.

8. Hay RJ. Tinea Capitis: Current Status. Mycopathologia. 2017;182(1-2):83-87.

9. García L, Espinosa J. Tiña capitis por Microsporum gypseum, una especie infrecuente. Arch Argent Pediatr. 2018;116(2):296-299.

10. Arenas R. Dermatophytosis. In: Arenas R, Estrada R, editors. Tropical dermatology. Georgetown: Landes; 2001: 4-11.

11. Reza A, Shokri H, Vahedi G. Factors in Etiology and Predisposition of Adult Tinea Capitis and Review of Published Literature. Mycopathologia. 2016;181;371-378.

12. Nenoff P, Krüger C, Ginter-Hanselmayer G, et al. Mycology-an update. Part 1 Dermatomycoses: Causative agents, epidemiology and pathogenesis. Journal of the German Society of Dermatology. $2014 ; 12(3) ; 188-210$. 
13. Verrier J, Monod M. Diagnosis of Dermatophytosis Using Molecular Biology. Mycopathologia. 2017;182(1-2):193-202.

14. Chiramel MJ, Sharma VK, Khandpur S, et al. Relevance of trichoscopy in the differential diagnosis of alopecia: A cross-sectional study from North India. Indian J Dermatol Venereol Leprol. 2016;82:651-658.

15. Chen X, Jiang X, Yang M, et al. Systemic antifungal therapy for tinea capitis in children. Cochrane Database Syst Rev. 2016;12:(5).
16. Arenas R, Toussaint S, Isa-Isa R. Kerion and dermatophytic granuloma. Mycological and histopathological findings in 19 children with inflammatory tinea capitits of the scalp. Inter $J$ Dermatol. $2006 ; 45(3): 215-219$ 